\title{
Frontières
}

\section{Le milieu communautaire et la prévention du suicide au Québec}

\author{
Vingt ans plus tard, où en sommes-nous ?
}

\section{Sylvaine Raymond}

Volume 12, numéro 1, automne 1999

Suicides, générations et culture

URI : https://id.erudit.org/iderudit/1074515ar

DOI : https://doi.org/10.7202/1074515ar

Aller au sommaire du numéro

Éditeur(s)

Université du Québec à Montréal

ISSN

1180-3479 (imprimé)

1916-0976 (numérique)

Découvrir la revue

Citer cet article

Raymond, S. (1999). Le milieu communautaire et la prévention du suicide au Québec : vingt ans plus tard, où en sommes-nous ? Frontières, 12(1), 89-93. https://doi.org/10.7202/1074515ar
Résumé de l'article

Le mouvement de la prévention du suicide au Québec trouve ses racines en Europe et plus particulièrement aux États-Unis. Devant l'augmentation des taux de suicide, plusieurs organismes communautaires québécois ont vu le jour grâce à l'implication bénévole de leur communauté : les Centres de prévention du suicide (CPS). Durant de nombreuses années, les CPS ont été le moteur du développement du mouvement de la prévention du suicide au Québec. En 1998, la Stratégie québécoise d'action face au suicide a été lancée. Cette Stratégie apporte un nouvel élan à la prévention du suicide mais comporte de nombreux défis. 


\section{Résumé}

Le mouvement de la prévention du suicide au Québec trouve ses racines en Europe et plus particulièrement aux États-Unis. Devant I'augmentation des taux de suicide, plusieurs organismes communautaires québécois ont vu le jour grâce à l'implication bénévole de leur communauté: les Centres de prévention du suicide (CPS). Durant de nombreuses années, les CPS ont été le moteur du développement du mouvement de la prévention du suicide au Québec. En 1998, la Stratégie québécoise d'action face au suicide a été lancée. Cette Stratégie apporte un nouvel élan à la prévention du suicide mais comporte de nombreux défis.

Mots clés: suicide - prévention - Québec - milieux communautaires

\section{Abstract}

The suicide prevention movement in Québec has its origin in Europe and more specifically in the United States. Faced with rising suicide rates, several community organizations in Québec came into existence thanks to the volunteer involvement from their community: The Centers for the Prevention of Suicide (CPS). For many years the CPS's have been the driving force behind the development of the suicide prevention movement in Québec. In 1998, la Stratégie québécoise d'action face au suicide (The Québec Suicide Prevention Task Force) was set up. This task force breathes new life into suicide prevention yet brings along with it a number of challenges.

Key words: suicide - prevention - Québec - community organizations

\section{Le milieu communautaire et la prévention du suicide au Québec Vingt ans plus tard, où en sommes-nous?}

\section{Sylvaine Raymond*, \\ directrice de l'Association québécoise de suicidologie.}

Le mouvement de la prévention du suicide au Québec date du milieu des années soixante-dix, ce qui est somme toute assez récent. À l'aube de l'an 2000, quel portrait pouvons-nous en faire? La venue de la Stratégie québécoise d'action face au suicide ${ }^{1}$ apporte-t-elle de nouvelles bases ou encore de nouvelles avenues pour les Québécois et les Québécoises? Quels sont les défis actuels à relever, tant pour les organismes communautaires que pour les organismes du réseau public et parapublic?

Pour répondre à ces questions, un bref aperçu de l'historique de la prévention du suicide, tant au Québec qu'en Europe et aux États-Unis, sera présenté. Par la suite, les principales activités de prévention du suicide initiées par les organismes communautaires seront brièvement décrites et commentées. Certains enjeux reliés à la Stratégie québécoise d'action face au suicide et à son implantation seront finalement discutés.

D'entrée de jeu, soulignons que la position présentée ici place les organismes communautaires au cœur du développement du mouvement de la prévention du suicide au Québec. Par contre, ce texte n'a pas la prétention de couvrir toutes les facettes de la prévention du suicide au Québec. Selon nous, le point de vue apporté ici illustre cependant avec honnêteté une démarche québécoise et suscite un questionnement actuel et présent.

\section{RAPPEL HISTORIQUE}

L'histoire de la prévention du suicide au Québec s'inscrit dans une démarche qui origine d'Europe et des États-Unis. Ainsi, au début du vingtième siècle, en Angleterre, l'Armée du salut ouvre un Bureau anti-suicide alors qu'à New York, le National Save-a-Life League prend naissance.

On ne peut traiter du développement de la prévention du suicide au Québec sans rappeler la naissance des «Samaritans» en 1953 en Angleterre. Le révérend Chad Varah ouvre alors un service pour les personnes suicidaires dans un sous-sol d'église et demande à des personnes bénévoles d'accueillir les arrivants, de leur servir le thé et les biscuits en attendant qu'il puisse les rencontrer. Quelle ne fut pas la surprise du révérend Varah de s'apercevoir que les bénévoles apportaient réconfort, écoute et conseils aux personnes qui venaient dans son local, et que les résultats de ces interventions bénévoles semblaient tout aussi pertinents que ses propres interventions plus cliniques. L'intervention bénévole, apportée par des personnes de la communauté, a alors été identifiée comme une nouvelle avenue pour aider les 
personnes en détresse. D'ailleurs, selon Dublin $^{2}$, l'intervention non professionnelle est sans doute une des découvertes les plus importantes dans le domaine de la prévention du suicide depuis les années 1930. Aujourd'hui, on retrouve plus de 200 organismes liés aux Samaritains dans le monde.

C'est en 1958, à Los Angeles, que naît le premier Centre de prévention du suicide, sous la direction de Norman Farberow, Edwin Shneidman et autres collaborateurs. Le Centre de prévention du suicide de Los Angeles offre alors des services d'intervention téléphonique, des activités de formation auprès des intervenants, et initie de nombreux projets de recherche. À l'instar des Samaritains, les services du Centre de prévention du suicide de Los Angeles sont offerts par des bénévoles, auxquels se joignent des professionnels.

Les travaux de Farberow et de Shneidman ont donné lieu à de nombreux écrits théoriques et à une compréhension différente du suicide. Shneidman ${ }^{3}$ indique que les personnes suicidaires partagent dix caractéristiques communes. Parmi ces caractéristiques citons, entre autres, une douleur psychologique intolérable, la recherche de la cessation de la conscience de cette douleur, le désespoir, l'ambivalence, la communication de l'intention. Les contributions de Shneidman et de ses collègues, tant au niveau de la compréhension du suicide qu'au niveau de la mise en place du premier centre de prévention du suicide, ont été des plus importantes pour le mouvement de la prévention du suicide en Amérique du Nord.

\section{AU QUÉBEC}

$\mathrm{Au}$ Québec, le problème du suicide inquiète et suscite diverses actions depuis plus d'une vingtaine d'année. Ainsi, en 1978, le ministère des Affaires sociales du Québec remet une première subvention à l'université Laval, ce qui a résulté en la mise en opération du premier CPS, le Centre de prévention du suicide de Québec. La fin des années 1970 est aussi l'époque de travaux d'envergure, comme l'étude épidémiologique de Marie-France Charron en 1981 et la consultation organisée au Québec et à l'étranger par le Comité de la Santé mentale du Québec. Cette consultation a mené à la publication de l'Avis sur la prévention du suici$d e$ en 1982, qui se voulait d'ailleurs «l'ossature d'une véritable politique de prévention du suicide» 4 .

De 1982 à 1990, devant l'augmentation des taux de suicide, face aux constats de réponses souvent mal adap- tées du réseau de la santé et des services sociaux, et suite à l'expérimentation du Centre de prévention du suicide de Québec, nous avons assisté au développement d'organismes communautaires œuvrant en prévention du suicide. Ce développement des ressources communautaires s'est fait grâce aux efforts considérables déployés par les communautés locales qui, avec des moyens précaires, ont mis en place des programmes de prévention du suicide dans leur milieu. Ces organismes communautaires, généralement appelés Centres de prévention du suicide (CPS) ont majoritairement donné la priorité, dans un premier temps, à la mise en place de services téléphoniques d'intervention en cas de crise suicidaire. De nombreuses autres activités se sont par la suite greffées à ce service. Ces activités sont décrites un peu plus loin.

Durant cette période (1982-1990), le nombre de ressources spécifiques pour la prévention du suicide (CPS) est passé de un à dix-neuf organismes. Depuis 1990 , ce sont douze nouveaux organismes qui ont vu le jour, portant le total à 31 organismes en 1998.

\section{CENTRES DE PRÉVENTION DU SUICIDE}

Quelles sont les caractéristiques d'un Centre de prévention du suicide? Quelle est leur conception du suicide, de la personne suicidaire et des stratégies à mettre en place?

Il est difficile d'offrir une réponse unique à ces questions. Cependant, certaines caractéristiques sont à signaler. Nous regrouperons ces caractéristiques en trois volets: approche et modèle théorique, intervention et éthique, et taille et structure organisationnelle.

\section{APPROCHE ET MODÈLE THÉORIQUE}

L'approche développée par les CPS est une approche multifactorielle et inclut dans sa compréhension les dimensions personnelles, sociales, familiales, environnementales et culturelles. Il n'y a donc pas ici de causalité unique mais plutôt une synergie de facteurs qui influencent, dans un sens positif ou négatif, le développement d'un état de crise suicidaire. Cette crise se déroule au cours d'un processus suicidaire. Tout au long de ce processus, la personne en état de détresse tentera par divers moyens et par ses stratégies d'adaptation habituelles, de retrouver un équilibre émotif et de diminuer cet état de souffrance intolérable. La cessation de la souffrance devient donc l'objectif recherché, et le suicide prend de plus en plus de place.
Selon Baechler' , suite à l'échec des stratégies utilisées, le suicide est alors perçu comme la seule solution.

Reprenant les énoncés de Shnei$\mathrm{dman}^{6}$, cette approche tient également compte de l'ambivalence de la personne en détresse, qui oscille entre la vision en tunnel d'un futur intolérablement souffrant, et le désir ardent d'une vie différente. De plus, bien que l'idée du suicide puisse être présente depuis plus ou moins longtemps, le passage à l'acte peut se produire très rapidement, suite à des événements déclencheurs. Enfin, la personne suicidaire communiquera, de manière plus ou moins subtile, son intention suicidaire.

\section{INTERVENTION ET ÉTHIQUE}

Cette approche implique un modèle d'intervention qui tient compte des caractéristiques de la personne suicidaire (la douleur psychologique intolérable, la recherche de la cessation de la conscience de cette douleur, le désespoir, l'ambivalence, la communication de l'intention). Ainsi, l'intervention téléphonique se doit d'offrir une réponse rapide, accessible et disponible. Cette intervention veut ouvrir le champ des alternatives, entendre la souffrance, briser l'isolement affectif de la personne, modifier la vision de tunnel, diminuer l'état de crise (tension et souffrance) et, bien sûr, empêcher le passage à l'acte. Contrairement aux multiples lignes d'écoute téléphonique, les interventions sont confidentielles mais non anonymes et, dans des situations de dangerosité élevée, font appel aux services ambulanciers et policiers. L'intervention se distingue également par son aspect plus directif. Des grilles d'intervention et d'évaluation d'urgence, des ententes précises avec les services d'urgence, des procédures de références font partie des outils des intervenants téléphoniques. Finalement, soulignons que chaque CPS a développé des normes déontologiques précises, connues et adoptées par les intervenants.

L'approche développée par les CPS a également des implications sur leurs autres activités. Partant du principe que tous peuvent agir au sein de leur réseau, les activités de sensibilisation destinées à l'ensemble de la communauté, et les divers programmes de prévention, insistent sur les points suivants: l'importance de la problématique du suicide, la reconnaissance des signes précurseurs, les possibilités d'intervention et l'accompagnement vers des ressources compétentes. 
TAILLE ET STRUCTURE

ORGANISATIONNELLE

Issus de communautés différentes, de nature locale, régionale ou encore suprarégionale, desservant des régions urbaines, rurales ou semi-rurales, disposant de ressources financières inégales et souvent peu comparables, les CPS n'ont pas tous la même structure, ni la même taille. Sous la responsabilité d'un conseil d'administration où siègent des individus de la communauté, les activités des CPS sont majoritairement le fait d'un petit nombre d'intervenants rémunérés (de 1 à 10) et d'intervenants bénévoles. Ces intervenants bénévoles proviennent de toutes les couches de la société et reçoivent une formation en intervention de crise suicidaire. La formation des bénévoles est d'une durée moyenne de 60 heures. De plus, les intervenants bénévoles sont encadrés et disposent de supervision clinique, de soutien et de formation continue.

Par contre, ce ne sont pas tous les CPS qui peuvent compter sur des intervenants bénévoles. La lourdeur de la problématique, la grande disponibilité demandée aux bénévoles et la faible densité de la population locale ont porté certains CPS à confier les interventions téléphoniques à des intervenants rémunérés.

\section{BILAN DES ACTIVITÉS DES CPS EN PRÉVENTION}

Parmi les diverses activités mises en place par les CPS pour prévenir le suicide au Québec, citons les services d'intervention cliniques, les activités de sensibilisation et de formation, le développement de programmes de prévention auprès de groupes à risque et la concertation entre les milieux.

\section{INTERVENTION CLINIQUE}

Nous avons décrit plus haut les interventions téléphoniques Outre cette description, soulignons que l'intervention téléphonique s'adresse tant à la personne suicidaire qu'à son entourage, aux personnes endeuillées suite à un suicide, et aux intervenants de la région. L'intervention téléphonique est également une porte d'entrée vers d'autres services: suivis à court et moyen terme, accompagnement, intervention auprès de la famille, références personnalisées, etc.

La majorité des régions peuvent actuellement compter sur un service d'intervention téléphonique. Cependant, ces services ne sont pas tous dispensés 24 heures par jour, à cause d'un sousfinancement notable. Finalement, soulignons que, dans certaines régions, les services d'intervention téléphonique sont dispensés par des ressources autres que des CPS: Info-santé, Centre de crise, etc. Quant aux interventions en face à face auprès des personnes suicidaires, elles sont moins répandues parce que les réalités géographiques les rendent souvent moins accessibles pour le client. Afin de contrer cette difficulté, des mécanismes de références avec d'autres organismes de la région sont établis; l'intervention téléphonique devient par la suite une intervention complémentaire du suivi.

À partir des années 1990, tout un nouveau champ d'intervention s'est implanté: l'intervention auprès des personnes endeuillées par suicide. Offrant des interventions individuelles, des interventions familiales, des groupes de soutien, des groupes d'entraide, ainsi que des activités dans divers milieux de vie (école, travail, etc.), les CPS ont ici encore innové et répondu à des besoins exprimés par leur communauté.

\section{SENSIBILISATION DE LA COMMUNAUTÉ ET CONCERTATION}

Dès le début des activités, les CPS ont adopté une approche qui insistait sur le rôle si important que tous les membres de la communauté peuvent jouer en prévention du suicide. Aussi, depuis 1980, d'innombrables activités de sensibilisation dans les communautés ont été mises sur pied. Ces activités de sensibilisation visent à outiller les personnes de la communauté pour dépister et agir rapidement auprès d'une personne suicidaire, tout en promouvant l'utilisation des ressources de la communauté. Cette sensibilisation s'est faite en même temps que l'établissement de liens de collaboration et de partenariat avec les diverses ressources des milieux. Ces milieux se sont pris en charge et ont, à leur tour, développé leur expertise dans leurs interventions auprès de leurs clients suicidaires.

\section{ACTIVITÉS DE FORMATION}

Les activités de formation ont pour objectif d'habiliter les intervenants de divers milieux à gérer un état de crise suicidaire. Cette gestion peut se limiter à reconnaître une situation de crise suicidaire, à en évaluer l'urgence et à accompagner la personne en détresse vers une ressource compétente. Si l'intervenant se sent suffisamment à l'aise, cette gestion inclut un plan d'intervention complet jusqu'à la résolution de l'état de crise.

$\mathrm{Au}$ fil des années, les organismes communautaires œuvrant en prévention du suicide ont largement contribué à la formation des personnes qui interviennent actuellement auprès des personnes suicidaires et de leur entourage et ce, dans le réseau scolaire, dans le milieu de la santé et des services sociaux, ainsi qu'au sein d'autres organismes.

Les CPS ont également élargi leurs champs d'action en développant des activités de formation touchant des clientèles plus spécifiques (personnes, familles et milieux endeuillés), ou encore en fonction de divers groupes d'âge (enfants, adolescents, aînés). Â ces formations se sont ajoutées des activités visant à apporter un soutien clinique aux intervenants, ainsi que de la formation continue.

DÉVELOPPEMENT DE PROGRAMMES

AUPRĖS DES GROUPES À RISQUE

Le Québec se distingue par un développement considérable de programmes de prévention du suicide auprès de groupes à risque. Au milieu des années 1980 , les premiers programmes de prévention du suicide auprès des jeunes, principalement en milieu scolaire, sont apparus. Les jeunes du niveau secondaire ont d'ailleurs été la cible la plus souvent identifiée dans les programmes de prévention du suicide 7 . D'autres groupes ont été ciblés par la suite. Citons les milieux carcéraux, les personnes âgées, les veufs, les personnes ayant fait des tentatives de suicide et, plus récemment, les hommes de 15 à 40 ans.

Ces programmes de prévention ont souvent été initiés par les CPS qui ont approché leurs partenaires locaux et régionaux. La diffusion de l'existence et de l'expérimentation de ces programmes, ainsi qu'une volonté tangible d'agir dans leur environnement, ont alors incité de nombreux milieux à développer à leur tour d'autres programmes de prévention.

\section{RAYONNEMENT DES CPS}

Depuis le début de la présente décennie, les activités en prévention du suicide ont connu un essor important. Plusieurs intervenants (bénévoles ou rémunérés) des CPS ont changé d'emploi et sont devenus, à leur tour, des acteurs importants en prévention du suicide dans leur nouveau milieu. De nombreux étudiants universitaires ont effectué des stages (recherche ou intervention) en milieu communautaire, et ont par la suite formé de nouveaux étudiants, tout en développant le champ de la recherche, influençant à leur tour le milieu communautaire. On peut donc parler ici d'interactions, de rétroactions 
permettant à tous de développer une pratique renouvelée.

La figure I illustre ces liens et le rayonnement qu'a permis le travail de pionnier des CPS.

\section{ET L'ÉTAT DANS TOUT CELA?}

Malgré le soutien financier progressif mais souvent minimal apporté par le gouvernement québécois à ces initiatives communautaires, le manque d'orientations générales et l'absence d'une priorité suicide ont longtemps porté à croire à un non-investissement de l'État dans ce dossier. Certains documents gouvernementaux semblent reposer sur les tablettes. Citons le projet de Politique de prévention du suicide (1986), le dossier Problématique du suicide, État des connaissances et perspectives de solutions, Direction de la Santé mentale, MSSS, (1990). De plus, jusqu'à la publication de la Politique de la Santé et du Bien-être (MSSS, 1992), les documents majeurs d'orientations, tels que le Rapport de la Commission Rochon (1987), le Rapport Harnois (1987) ou la Politique de santé mentale du MSSS (1989) laissent peu de place à la problématique du suicide, qui est souvent incluse dans le contexte très général de la «santé mentale». En 1990, l'Association québécoise de suicidologie ${ }^{8}$ réagissait à cette situation et présentait au MSSS un mémoire ${ }^{9}$ afin que le Gouvernement du Québec mette en place et opérationnalise un modèle québécois intégré de prévention du suicide. Par la suite, chaque événement public a été l'occasion de dire qu'il n'y avait pas de position ou de politique provinciale en regard de la prévention du suicide.

En 1992, le MSSS publie la Politique de la santé et du bien-être où le suicide est identifié comme l'objectif 17 qui vise à réduire de $15 \%$ le nombre de suicides et de tentatives de suicide d'ici l'an 2002. Cette politique donne également lieu à la décentralisation des pouvoirs et à la mise en place des Régies régionales.

Lors de sa conférence de presse dans le cadre de la Semaine provinciale de prévention du suicide en 1997, l'AQS réitérait publiquement sa demande pour que le gouvernement québécois se prononce et agisse. C'est à cette occasion que madame Colin, sous-ministre à la Santé publique, a annoncé la mise en place d'un groupe de travail provincial qui a réalisé la Stratégie québécoise d'action face au suicide. Enfin, les intervenants québécois pourraient être soutenus par une position et des orientations ministérielles.

\section{Figure 1}

\section{Le rayonnement des CPS}

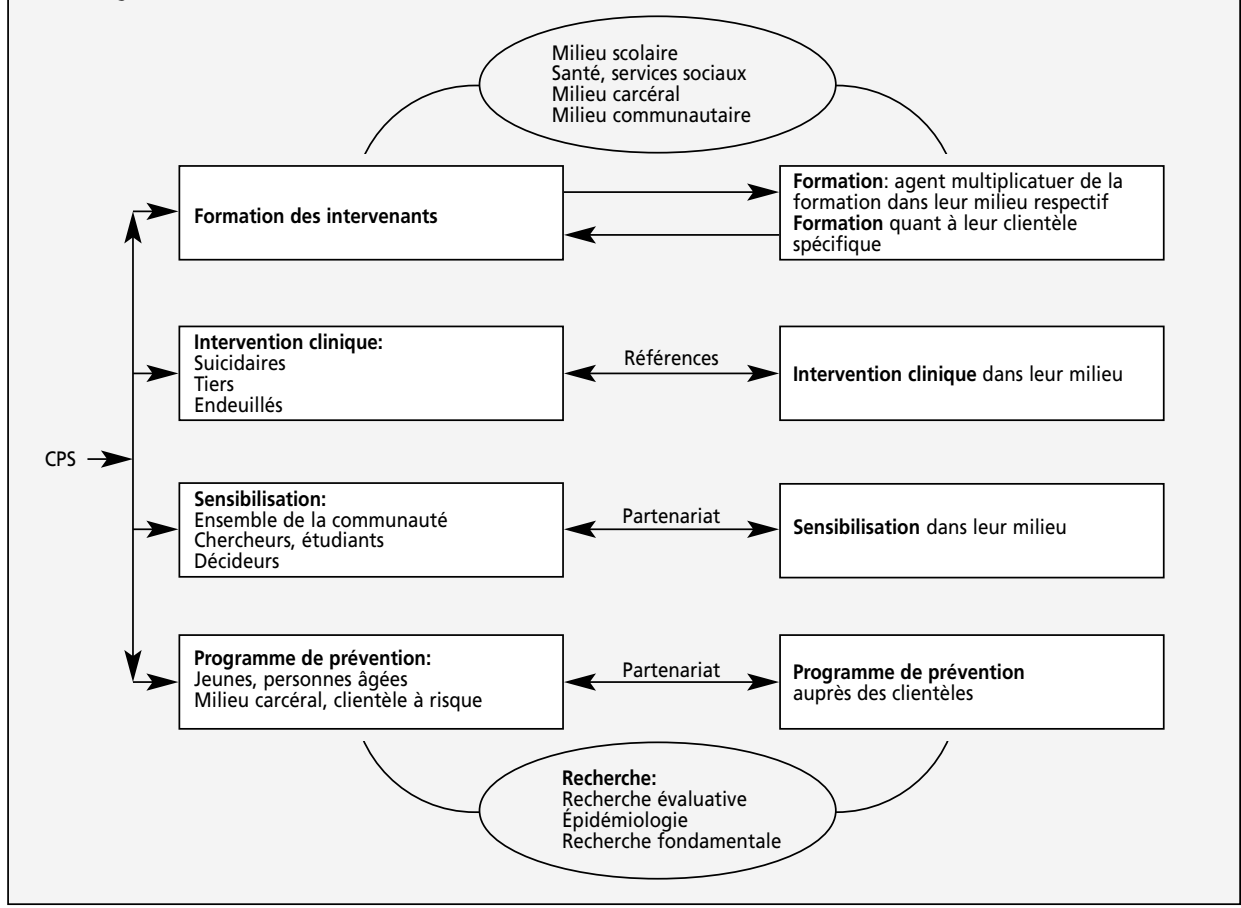

La Stratégie québécoise d'action face au suicide a été lancée en février 1998. Cette stratégie propose des actions, et identifie des priorités qui sont peu contestées de la part de l'ensemble des acteurs en prévention du suicide au Québec. La Stratégie reconnaît le rôle de «leader» jusqu'à maintenant joué par les organismes communautaires spécifiques et invite tous les organismes des divers réseaux à une mise en commun des efforts et à un réel partenariat en intervention et en prévention du suicide.

Cependant, cette stratégie est-elle la réponse pour contrer le suicide au Québec? Bien que peu de personnes en critiquent le contenu, certains éléments de sa mise en œuvre questionnent et interpellent. De nombreux défis sont à relever et certains méritent d'être soulignés.

\section{DÉFIS LIÉS À L'IMPLANTATION DE LA STRATÉGIE}

FINANCEMENT

$\mathrm{Au}$ moment du lancement de la Stratégie québécoise d'action face au suicide, le gouvernement annonçait un investissement de 700 000\$ réparti sur trois années financières, soit de 1998 à 2001. Par contre, ce financement est dédié à la recherche et au développement pour les groupes identifiés les plus à risque de suicide.

Sans nier l'importance de la recherche, la Stratégie a peu de chance de succès si elle n'est pas accompagnée d'un financement adéquat. À titre d'exemple, un des éléments centraux de la Stratégie est d'assurer une gamme essentielle de services dans toutes les régions du Québec: une intervention téléphonique $24 / 7$, une évaluation de la situation, un suivi et des protocoles de références entre les organismes. De plus, la majorité des intervenants doivent être en mesure de dépister une personne suicidaire et d'offrir minimalement une première intervention. Sans l'injection d'argent neuf lié à la mise en place de la gamme essentielle de services, on se retrouve dans une situation qui consiste pour tous à «en faire plus avec moins». Mentionnons également que dans un contexte de rationalisation budgétaire, de coupures, la nécessaire concertation risque d'être partiellement atteinte. Se concerter, c'est prendre le temps de se connaître, de développer des interventions communes et complémentaires. Or, avec des budgets restreints, plusieurs organismes se retrouvent à devoir faire des choix difficiles entre répondre à la communauté en demande ou s'engager dans le développement de stratégies locales ou régionales.

Par contre, au moment où ces quelques lignes sont écrites (avril 1999), le ministre délégué à la Santé et aux Services sociaux, monsieur Gilles Baril, annonçait une injection d'argent neuf de l'ordre de 3,5M\$. Bien que les besoins financiers des CPS et des autres organismes du réseau de la santé et des services sociaux soient largement supérieurs à ce montant, cet ajout viendra sans aucun doute soutenir les actions en prévention du suicide. 
«LEADERSHIP»

La Stratégie québécoise d'action face au suicide identifie les régies régionales comme étant responsables de son implantation dans leur région respective. Ainsi, selon les priorités régionales, les pressions de la population, l'intérêt des dirigeants, la prévention du suicide sera plus ou moins à l'agenda des priorités régionales. Fait aussi inquiétant, non seulement les décisions sont régionalisées mais elles sont ensuite re-découpées en territoire de CLSC ou de MRC. En multipliant les lieux de décisions, il y a des risques certains d'assister à des actions moins concertées avec des partenaires qui ont un mandat régional et non local. Est-ce réaliste de demander à un organisme régional ou suprarégional de participer à des rencontres et à des groupes de travail dans chacun des territoires qu'il dessert? Or, pour que la Stratégie prenne tout son sens et atteigne les objectifs fixés, toutes les régions du Québec doivent s'impliquer et suivre des lignes directrices claires.

Un autre leadership important est le leadership provincial. Bien que la Stratégie prévoie une forme de comité provincial de suivi à l'implantation, le seul comité qui a été mis en place est un comité MSSS-Régies, qui vise à faire circuler l'information entre les régies et à développer de nouveaux projets. Or, un vrai comité provincial devrait réunir tous les organismes provinciaux interpellés par la Stratégie (ministère de l'Éducation, représentant du milieu hospitalier, du milieu de la justice, des CLSC, etc). Ce comité pourrait devenir un lieu central d'information, de références, de partenariat, de priorisation et d'orientations conjointes. En ce moment, nous assistons à une multitude d'actions et de mises en place de comités qui se réunissent en vase clos, de groupes de travail qui dédoublent les activités et les plans d'actions, réinventent la roue, méconnaissent leurs partenaires et luttent pour des pouvoirs ou encore pour une expertise à développer. La population du Québec aurait beaucoup à gagner de la mise en place d'un comité provincial, représentatif des forces vives en prévention du suicide.

\section{PARTENARIAT}

Un des aspects importants lié au défi $\mathrm{du}$ partenariat est celui de conjuguer positivement les interventions de plusieurs partenaires qui ont des interprétations différentes de l'étiologie du suicide, des langages différents et qui ne possèdent pas les mêmes ressources pour s'engager dans un processus de partenariat.
«TOUT LE MONDE PEUT INTERVENIR»

Avec la venue de la Stratégie, les gestionnaires des organismes concernés par l'implantation de la Stratégie confient facilement à un employé de leur organisation un mandat spécifique d'intervention auprès de la clientèle qui présente une problématique suicidaire, sans nécessairement s'assurer au préalable de l'intérêt et des habiletés de cet employé. Intervenir auprès d'une personne suicidaire implique certes des compétences au niveau du "savoir-faire» mais surtout au niveau du "savoir-être». Ce savoirêtre se développe à partir d'une réflexion approfondie sur les limites personnelles de l'intervenant, sur ses attitudes et sur ses croyances. Ce ne sont pas tous les intervenants qui sont à l'aise avec une intervention auprès des personnes suicidaires. De plus, le développement du savoir-faire nécessite une masse critique d'intervention, masse critique qui ne se retrouve vraiment pas partout. Techniquement, tout le monde peut intervenir, ne serait-ce qu'en accompagnant la personne vers une ressource compétente. Cependant, il y aurait avantage à définir cette intervention et à s'assurer qu'elle soit le fait d'intervenants adéquatement formés et soutenus.

\section{ET LA PLACE DU COMMUNAUTAIRE DANS TOUT CELA?}

Après avoir été un moteur pour le mouvement de la prévention du suicide au Québec, les CPS se retrouvent, de façon générale, à titre de partenaire comme les autres dans une approche qui vise à impliquer tous les acteurs. Leur rôle en tant qu'organisme spécialisé et de point central a été modifié pour devenir celui d'un organisme communautaire sur lequel on compte, mais qui n'est pas nécessairement privilégié. Pourtant, voilà des années que les CPS voulaient travailler en lien avec les partenaires et qu'ils clamaient haut et fort que la prévention du suicide est l'affaire de tout le monde, que tous les partenaires devaient jouer un rôle, en fonction de leurs spécificités. La Stratégie, et son implantation, porte tous les acteurs à s'impliquer dans des actions de prévention et d'intervention. Cette appropriation récente de la problématique par de nombreux organismes s'accompagne d'interventions isolées, non concertées. On assiste presque à une lutte pour une expertise reconnue par les pairs. Espérons que tous verront l'importance, pour la personne en détresse, de travailler de concert afin d'atteindre un objectif commun.

\section{Notes}

* L'auteure tient à souligner que ce texte est un point de vue personnel et ne représente pas nécessairement la position officielle de l'Association québécoise de suicidologie.

1 MINISTÈRE DE LA SANTÉ ET DES SERVICES SOCIAUX, S'entraider pour la vie, Stratégie québécoise d'action face au suicide, Direction de la santé publique, 1998.

2 Louis I. DUBLIN, «Suicide Prevention» dans Edwin S. SHNEIDMAN, On the Nature of Suicide, San Francisco, American Association of Suicidology, 1987.

3 Edwin S. SHNEIDMAN, Definition of Suicide, New York et Toronto, J. WileyInterscience Publication, 1985.

4 ASSOCIATION QUÉBÉCOISE DE SUICIDOLOGIE, La Prévention du suicide au Québec: vers un modèle intégré de services, novembre 1990.

5 J. BAECHLER, Les Suicides, Paris, Calmann-Levy, 1981.

6 Edwin S. SHNEIDMAN, ibid.

7 J.J. BRETON et al., Bilan des recherches évaluatives sur les programmes d'intervention et de prévention du suicide chez les jeunes au Canada, Québec, Hôpital Rivières-des-Prairies, 1998.

8 L'Association québécoise de suicidologie est un organisme communautaire provincial dont la mission est la promotion de la prévention $\mathrm{du}$ suicide au Québec. L'isolement des intervenants et des CPS, ainsi que la volonté de rassembler les forces, de faire avancer les connaissances et de défendre des dossiers prioritaires ont contribué à la naissance de l'Association québécoise de suicidologie. Pendant les sept premières années de son existence, l'AQS a fonctionné grâce au travail bénévole des membres des conseils d'administration et de divers comités. Les colloques provinciaux, les Semaines provinciales de prévention du suicide, les prises de position ont donc également fait avancer la cause de la prévention du suicide au Québec.

9 ASSOCIATION QUÉBÉCOISE DE SUICIDOLOGIE, La Prévention du suicide au Québec: vers un modèle intégré de services, novembre 1990. 